\title{
THE EFFECT OF ALKALINE TREATMENT ON THE PROPERTIES OF HIERARCHICAL ZSM-5 PREPARED BY POST-SYNTHESIS DESILICATION
}

\author{
D. Hartanto ${ }^{*}$, D. O. K. Wardhana, W. P. Utomo and Y. L. Ni'mah \\ Department of Chemistry, Faculty of Sciences, \\ Institut Teknologi SepuluhNopember (ITS), 60111, Indonesia \\ *E-mail: djokohar@chem.its.ac.id
}

\begin{abstract}
The effect of alkaline treatment on properties of hierarchical ZSM-5 was investigated in this work. The ZSM-5 zeolite was treated by aqueous solution of $\mathrm{NaOHwith}$ variation concentration of $0.25,0.5,1.0$ and $2.0 \mathrm{M}$. The properties of hierarchical ZSM-5 were characterized by XRD, FTIR, SEM, and isothermal $\mathrm{N}_{2}$-sorption. The XRD analysis shows that all samples consist of ZSM-5 and tridymite phase. The $\mathrm{NaOH}$ treatment doesn't have a significant effect on the structural phase but greatly influence the crystallinity. The higher $\mathrm{NaOH}$ concentration, the lower the relative crystallinity. FTIR analysis confirms the presence of ZSM-5 structure without any new bonding formation. SEM examination revealed that $\mathrm{NaOH}$ treatment could change the morphology of the particles indicatedby the formation ofthe hole and crack in the ZSM-5 particle's surface as a result of desilication. The $\mathrm{N}_{2-}$ sorption shows that increasing $\mathrm{NaOH}$ concentration can reduce the particle's surface area. The treatment succeeds to

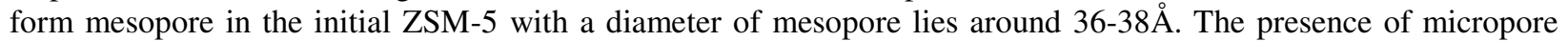
and mesopore in all samples confirms the formation of hierarchical ZSM-5 after alkaline treatment using $\mathrm{NaOH}$ solution.
\end{abstract}

Keywords: ZSM-5, Desilication, Hierarchical, NaOH Treatment, Mesopore

(C) RASĀYAN. All rights reserved

\section{INTRODUCTION}

ZSM-5 is recently becoming one of the zeolite families that attract many researchers due to its unique acidity and well-defined microporosity. ZSM-5 has excellent heterogeneous acidity, great thermal and mechanical stability, well shape selectivity and interconnected two-dimensional micropore system which contribute to the usage as a catalyst in petroleum and petrochemical industry ${ }^{1-3}$. However, the microporous nature of ZSM-5 imposes diffusion limitations for reaction or adsorption process, especially for large molecules. The mass-transfer limitation indirectly affects the catalytic activity, selectivity and lifetime of ZSM- $5^{4}$. Based on these facts, it is desirable to improve the porosity properties of ZSM-5for various applications. The properties of ZSM-5 could be enhanced by the formation of mesopore in the ZSM-5 particles. This formation of mesoporosity in ZSM-5, that naturally has micropore only, produces the hierarchical ZSM-5. The mesopore could enlarge the particle's surface area and shorter intracrystalline path length ${ }^{5}$. Moreover, this mesopore could optimize the diffusion of large molecules to reach the active site in the $\mathrm{ZSM}-5^{6}$.

The formation of mesopore in ZSM-5 resulting hierarchical mesoporous ZSM-5 could be carried out by using bottom-up or top-down process. The bottom-up process is carried out when mesoporosity was introduced to the ZSM-5 particle along with the synthesis process, for example by addition of organic structure directing agent (OSDA). In the other hand, the top-down process involves post synthesis treatment to the previous synthesized ZSM-5 by desilication and/or dealumination process ${ }^{7}$. However, the top-down process is usually applied for preparing mesoporous ZSM-5 as this process is easier to be conducted rather than bottom-up process. Due to its simplicity, the top-down process is also more visible to be conducted in large scale application. One of the most interesting processes in the top-down process is desilication of zeolites using an alkaline solution such as $\mathrm{NaOH}$ or $\mathrm{KOH}$, or a weak organic base such Rasayan J. Chem., 12(2), 939-946(2019)

http://dx.doi.org/10.31788/RJC.2019.1225142

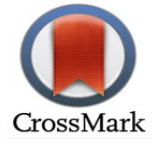


as tetrapropylamonium hydroxide $(\mathrm{TPAOH})^{8}$. This process could generate larger pore in zeolites particle resulting in hierarchical zeolites. In our case that ZSM-5 naturally has micropores only, the desilication could initiate the formation of mesopore or even macropore. This process could also form the interconnected mesopore system in the particle of zeolite thus increase the diffusion rate of the molecules to the zeolites particles ${ }^{6,9}$.

The desilication process using $\mathrm{NaOH}$ solution on several zeolites has been reported by several researchers. Matias et al. (2011) reported that the desilication of TON zeolite $(\mathrm{Si} / \mathrm{Al}=31)$ using $\mathrm{NaOH}$ with different concentration could create the mesopores with pore diameter about $20 \mathrm{~nm}$. However, this alkaline treatment also gave a slight decrease in Brönsted acidity but a significant increase in Lewis acidity. Unfortunately, this desilication did not have influence in the catalytic activity ${ }^{10}$. Sadowska et al. (2013) also reported that the desilication of highly siliceous ZSM-5 using $\mathrm{NaOH}$ solution could be more effective in the surface zone of the particle than in the bulk. They also revealed that the increase of temperature used for desilication process could increase the extraction of $\mathrm{Si}$ as well as Al thus it could produce increasing of the volume and surface of the mesopore ${ }^{11}$.

Those previous reports show that desilication is also potential to be further developed to prepare mesoporous ZSM-5. The further understanding of the alkaline solution parameter and its effect on the mesoporosity is still also crucial. Thus, in recent study, we focus on the effect of $\mathrm{NaOH}$ concentration to the mesoporosity and properties of ZSM-5. The concentration could influence the amount of Si that is extracted from ZSM-5 so it also influences the formation of mesopore in the ZSM-5 particles. The process of desilication process and their effect is discussed in detail.

\section{Material and Methods}

\section{EXPERIMENTAL}

The materials used in this study were $\mathrm{NaOH}$ pellets (Merck, Germany), commercial ZSM-5 (Pingxiang Naike Chemicals, China), Whatmann No.40 filter paper, ammonium acetate (Merck, Germany) and oxalic acid (Merck, Germany). Aqua DI (deionized water) was taken from a Millipore Milli-Q system and used within 1 week.

\section{General Procedure}

\section{Parent ZSM-5 Preparation}

The commercial ZSM-5 was directly used without further purification. Prior to modification of ZSM-5 with the post-synthesis desilication process, the commercial ZSM-5 was dried at $105{ }^{\circ} \mathrm{C}$ for 1 hour inthe oven to remove excessive adsorbed water. The samples were then characterized using XRD to analyze the phase and crystallinity of commercial ZSM-5.

\section{Desilication of ZSM-5 by Alkaline Treatment}

Modification of hierarchical ZSM-5 with alkalinedesilication was started by stirring $\mathrm{NaOH}$ solution at $500 \mathrm{rpm}$ and heating to a constant temperature of $65^{\circ} \mathrm{C}$. The variation of $\mathrm{NaOH}$ concentration used was $0.25 ; 0.5 ; 1$; and $2 \mathrm{M}$. After stirring, ZSM-5 was put into $\mathrm{NaOH}$ solution for 30 minutes with solid and liquid ratio of $33 \mathrm{~g} / \mathrm{L}$. After 30 minutes of stirring, the mixture of ZSM-5 and $\mathrm{NaOH}$ solution was quenched by cooling the mixture in ice water bathto reach room temperature. After that, the solution was filtered using Whatmann number 40 filter paper and washed using aqua DI. The washing process is repeated 3 times. Then,the obtained ZSM-5 was dried at $105{ }^{\circ} \mathrm{C}$ for 12 hours. The resulted product was then ion exchanged to form H-ZSM-5. This ion exchange process was carried out bymixing ZSM-5 with $\mathrm{NH}_{4} \mathrm{CH}_{3} \mathrm{COO} 1 \mathrm{~N}$ solution under stirring and refluxed at a constant temperature of $60{ }^{\circ} \mathrm{C}$ for 3 hours. The solid and liquid ratio for this process was $1 \mathrm{~g}$ over $20 \mathrm{~mL}$ of solution. ZSM-5 was then filtered and washed with aqua DI. ZSM-5 was dried in the oven with the temperature of $105{ }^{\circ} \mathrm{C}$ for 12 hours. The ZSM-5 was then calcined at $550{ }^{\circ} \mathrm{C}$ for 6 hours. The ZSM-5 that has been desilicated and cation exchanged is subsequently called ZSM-5-X with $\mathrm{X}$ was $\mathrm{NaOH}$ concentration.

\section{Characterization of ZSM-5}

The phase purity and crystallinity of ZSM-5 was analyzed using powder X-ray diffraction (XRD) PANalytical X'Pert MPD. The sample was placed in the sample holder. The X-ray irradiation was carried out using CuKa irradiation ( $\lambda=1.54056 \AA$ ) at $2 \theta$ of 20 to $100^{\circ}$ with a step size of $0.1^{\circ}$. 
RASĀYAN J. Chem.

Vol. 12 | No. 2 |939 - 946| April - June | 2019

The crystallinity of the samples was analyzed using Equation 1. The highest peak is used to calculate this relative intensity. The $I_{\text {sample }}$ is the intensity of the analyzed sample, and $I_{\text {reference }}$ is the intensity of the reference sample.

$$
\text { Relativeintensity }=\frac{I_{\text {sample }}}{I_{\text {reference }}} \times 100 \%
$$

The Infrared analysis was performed to analyze the functional group of ZSM-5. This analysis is performed using Shimadzu Instrument Spectrum One 8400S on fingerprint area.

Scanning Electron Microscopy (SEM) was used to examine the morphological properties of ZSM-5. The analysis was carried out using ZEISS EVO MA. The sample was placed on carbon tape and coated with gold-palladium to increase the conductivity. The imaging process was performed using the Secondary Imaging (SE) detector.

The $\mathrm{N}_{2}$-sorption was carried out to analyze the specific surface area and porosity of resulted ZSM-5. The analysis was performed using Micromeritics ASAP 2020. The analysis of specific surface area was calculated using Brunauer-Emmett-Teller (BET) adsorption isotherm model, while the analysis of pore diameter was calculated using Barrett-Joyner-Halenda (BJH) model.

\section{X-Ray Diffraction (XRD)}

\section{RESULTS AND DISCUSSION}

The XRD characterization is performed to analyze the phases that exist in the sample and the crystallinity as well. The diffractogram of the five samples is presented in Fig.-1. From Fig.-1, it is clear that all samples have, indeed, the typical peaks of ZSM-5 as indicated by the presence of peaks at $2 \theta=7.84$; $8.78 ; 22.98 ; 23.20 ;$ and $23.87^{\circ}$. However, all samples also exhibit additional peaks coming from another phase. Analysis of this additional phase shows that the peaks come from tridymite phase as indicated by peaks at $2 \theta=20.51 ; 21.67 ; 23.24 ; 30.02 ; 35.74$; and $35.98^{\circ}$. This result indicates that all samples consist of two phases, i.e. ZSM-5 and tridymite, where the main phases are still ZSM-5 because the characteristic peaks of ZSM-5 are the major peaks. The comparison between the characteristic peaks of ZSM-5 and tridymite is tabulated in Table-1 to ensure this finding.

Table-1: Characteristic Peaks of ZSM-5 and Tridymite

\begin{tabular}{c|c|c}
\hline Samples & $\begin{array}{c}\text { ZSM-5 } \\
(\text { PDF No. 96-150-5107) }\end{array}$ & $\begin{array}{c}\text { Tridymite } \\
(\text { PDF No.96-901-3494) }\end{array}$ \\
\hline ZSM-5 & $7.97 ; 8.83 ; 23.00 ; 23.20 ; 23.80$ & $20.76 ; 21.70 ; 23.20 ; 29.94 ; 36.07$ \\
\hline ZSM-5-0.25 & $7.87 ; 8.74 ; 22.93 ; 23.16 ; 23.79$ & $20.71 ; 21.64 ; 23.16 ; 29.90 ; 35.55 ; 39.95$ \\
\hline ZSM-5-0.5 & $7.89 ; 8.78 ; 22.77 ; 23.00 ; 23.63$ & $20.60 ; 21.41 ; 23.40 ; 30.10 ; 35.41 ; 35.79$ \\
\hline ZSM-5-1 & $7.81 ; 8.76 ; 22.92 ; 23.19 ; 23.82$ & $20.74 ; 21.57 ; 23.19 ; 30.30 ; 35.91$ \\
\hline ZSM-5-2 & $7.79 ; 8.74 ; 22.89 ; 23.14 ; 23.77$ & $20.66 ; 21.55 ; 23.14 ; 29.78 ; 35.89$ \\
\hline
\end{tabular}

Figure-1 also shows the difference in intensity between the six samples. In a certain amount of $\mathrm{NaOH}$ concentration, the crystallinity of the samples is higher than the untreated (commercial) one. The calculation of relative crystallinity is presented in Table-2. The highest relative crystallinity (as much as $133.230 \%$ ) appears when the ZSM-5 was treated with $0.25 \mathrm{M}$ of $\mathrm{NaOH}$. This crystallinity is slightly lower reaching $119.592 \%$ when the concentration is increased up to $0.5 \mathrm{M}$. However, these two concentrations still exhibit higher relative intensity than the untreated one. It might be caused by the reduction of tridymite intensity because the $\mathrm{Si}$ contained in the tridymite was also dissolved into $\mathrm{NaOH}$ solution. Tridymite was also a phase that contains a high amount of silica. The reduction of tridymite intensity affects the relative crystallinity of ZSM-5.

The increasing of $\mathrm{NaOH}$ concentration reduces significantly the relative crystallinity of the ZSM-5 as shown in ZSM-5-1 and ZSM-5-2. This reduction is caused by the dissolution of Si from the framework of ZSM-5 into NaOH solution. This Si dissolution occurs in the ZSM-5 surface that in contact with $\mathrm{NaOH}$ solution thus the crystal lattice of the ZSM-5 is still maintained ${ }^{4,9,12}$. The dissolution of Si is predicted to form the additional pores without changing in the ZSM-5 crystal structure as shown by XRD result. Similarly, Li et al. (2014) previously reported that the using of $\mathrm{NaOH}$ concentration of $0.1-0.3 \mathrm{M}$ did not 
RASĀYAN J. Chem.

Vol. 12 | No. 2 |939 - 946| April - June | 2019

change the crystallinity of zeolites, but when the concentration is higher, the crystallinity would be reduced due to partial dissolution of zeolite particles $^{12}$.

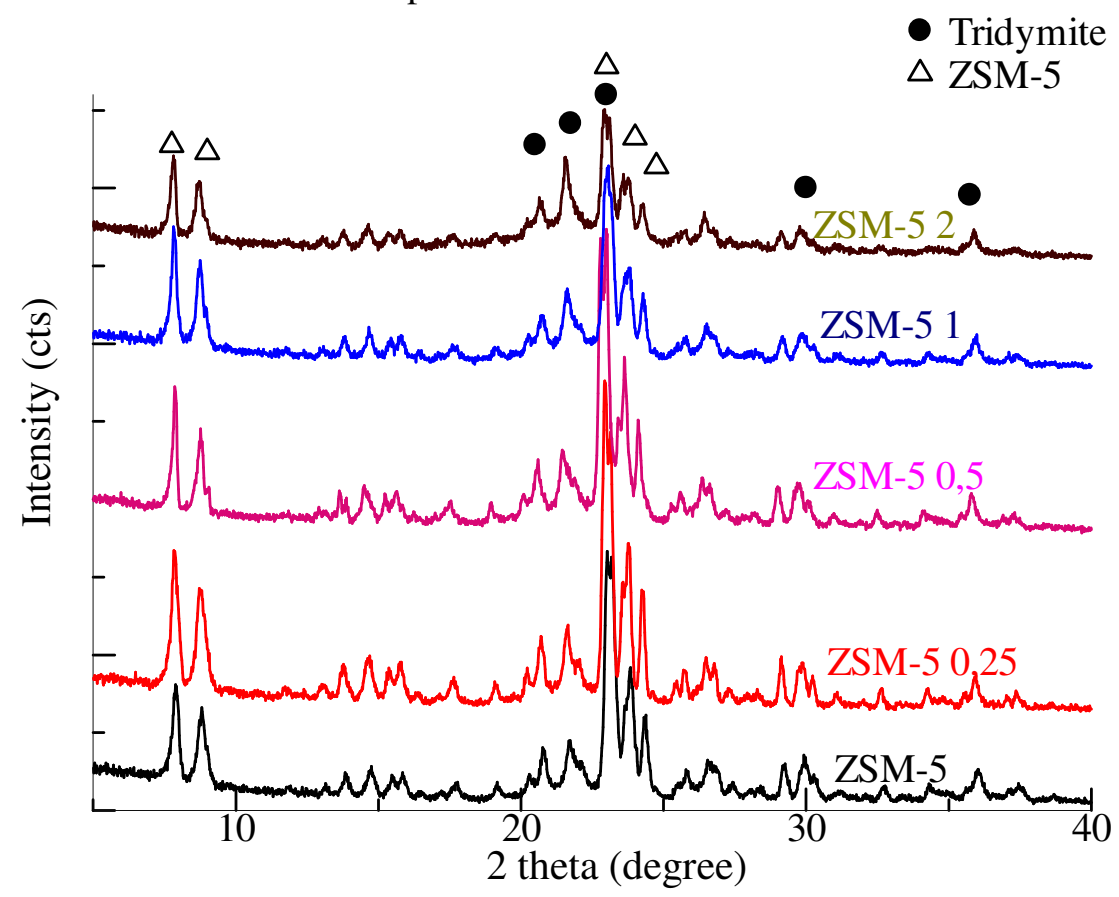

Fig.-1:Diffractogram of Commercial ZSM-5 and Hierarchical ZSM-5

Table-2:Relative Crystallinity of Commercial ZSM-5 and Hierarchical ZSM-5

\begin{tabular}{c|c}
\hline Materials & Relative Crystallinity $(\%)$ \\
\hline ZSM-5 & 100 \\
\hline ZSM-5-0.25 & 133.230 \\
\hline ZSM-5-0.5 & 119.592 \\
\hline ZSM-5-1 & 69.5103 \\
\hline ZSM-5-2 & 57.8365 \\
\hline
\end{tabular}

\section{Fourier Transform InfraRed (FTIR)}

Figure-2 shows the FTIR spectra of the commercial ZSM-5 and hierarchical ZSM-5 after desilication process. There are absorption bands in wave number of 450 and $550 \mathrm{~cm}^{-1}$ which indicate the presence of ZSM-5 crystals with MFI structure. This result in accordance with the previous report ${ }^{13,14}$. The absorption band at $550 \mathrm{~cm}^{-1}$ refers to the vibration of the pentacyl skeleton on the zeolites. The absorption band at wave number $450 \mathrm{~cm}^{-1}$ shows the vibration of T-O-T bending as reported by Abello et al. ${ }^{4}$ In addition, the absorption band at wave numbers between $1120-1070 \mathrm{~cm}^{-1}$ and $1230-1070 \mathrm{~cm}^{-1}$ show the vibration associated with asymmetric T-O-T stretching vibration. The absorption band at $800 \mathrm{~cm}^{-1}$ refers to the symmetric stretching vibration of T-O-T ${ }^{12-14}$. These absorption peaks are tabulated in Table- 3 .

The IR spectra show that there is not formation of new functional groups as indicated by the absence of new bond formation during the desilication process. The desilication process is only a process of dissolving silica inside and outside the ZSM-5 crystals. The results of ZSM-5 FTIR analysis showed that the difference in $\mathrm{NaOH}$ concentration does not cause the formation of new functional groups or chemical bonds in the hierarchical ZSM-5.

\section{Scanning Electron Microscopy (SEM)}

Figure-3 shows the SEM micrograph of the commercial ZSM-5. As shown in the Fig.-3, it seems that there are several irregular particles besides the typical ZSM-5 particles. The typical ZSM-5 particles are usually indicated by the hexagonal formed particle. These irregular particles are possibly the particles from the tridymite that exist as an impurity. Surprisingly, the impurities phases of tridymite were 
RASĀYAN J. Chem.

Vol. 12 | No. 2 |939 - 946| April - June | 2019

diminished by the addition of alkaline concentration as shown in Fig.-4. This is likely due to the dissolution of $\mathrm{Si}$ by $\mathrm{NaOH}$ solution.

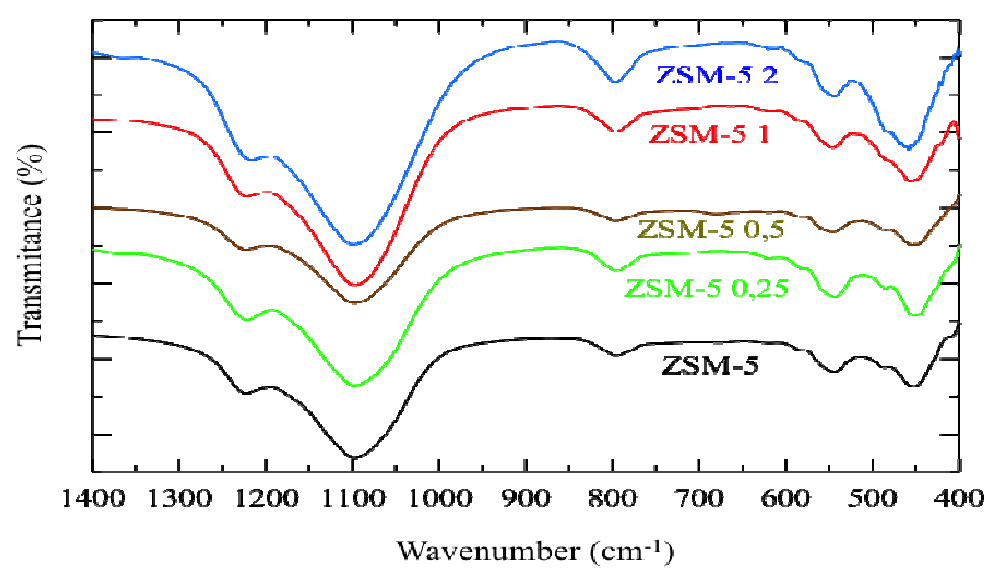

Fig.-2: IR Spectra of Commercial and Hierarchical ZSM-5

Table-3: Spectra IR Data of Commercial and Hierarchical ZSM-5

\begin{tabular}{c|c|c|c|c|c}
\hline \multicolumn{5}{|c|}{ Wavenumber } & Functional Group \\
\hline ZSM-5 & ZSM-5-0.25 & ZSM-5-0.5 & ZSM-5-1 & ZSM-5-2 & 1217.1 \\
\hline 1222.9 & 1220.9 & 1222.9 & 1222.9 & $\begin{array}{c}\text { asymmetric T-O-T stretching } \\
\text { vibration }\end{array}$ \\
\hline 1097.5 & 1097.5 & 1097.5 & 1097.5 & 1099.4 & $\begin{array}{c}\text { asymmetric T-O-T stretching } \\
\text { vibration }\end{array}$ \\
\hline 796.63 & 796.63 & 796.63 & 796.63 & 796.63 & $\begin{array}{c}\text { symmetric T-O-T stretching } \\
\text { vibration }\end{array}$ \\
\hline 543 & 543.94 & 545.87 & 545.87 & 545.87 & $\begin{array}{c}\text { vibration of the pentacyl ZSM- } \\
5\end{array}$ \\
\hline 451 & 449.43 & 449.43 & 455.22 & 459.07 & T-O-T bending vibration \\
\hline
\end{tabular}

The $\mathrm{NaOH}$ treatment on ZSM-5 particle shows the formation of holes and crack in the surface of particles which are getting more massive until the side of the particles are eroded. ZSM-5-0.25 begins to experience slight damage on their particles. However, the irregular particles also begin to be diminished indicating the removal of impurity's particle. The higher $\mathrm{NaOH}$ concentration applied, the higher morphology reduction of ZSM-5 particles and the higher removal of irregular particles (impurity's particles). At the concentrations greater than 0.5, it seems that the destruction of ZSM-5 particles is more massive ZSM-5 as indicated by the eroded walls of the ZSM-5 particles. This result is in good accordance with the XRD result that shows the lowest relative crystallinity. The result is also in accordance with withKhoshbin andKarimzadeh(2017) that alkali treatmentcould damagethe morphology of ZSM-5 particles ${ }^{15}$.

\section{$\mathbf{N}_{\mathbf{2}}$-Sorption}

Figure-5 shows the BET isothermal curve of the commercial and hierarchical ZSM-5. Table-4 shows that the increasing $\mathrm{NaOH}$ concentration decreases the BET surface area $\left(\mathrm{S}_{\mathrm{BET}}\right)$ of the particles. This decreasing of the surface area is predicted as a result of micropore damage to form mesopores. Increasing the specific surface area of BET (SBET) in ZSM-5-0.25 confirms the dissolution of Si in tridymite and ZSM-5 phases so that it can increase the BET surface area. However, the surface area of ZSM-5-0.5; ZSM-5-1; and ZSM-5-2 decreases. This is caused by the damage of ZSM-5 crystals and an increasing number of mesopores after treatment with more concentrated $\mathrm{NaOH}$ solution. This result is in good accordance with the SEM image in Fig.-4 which shows that the damaging of ZSM-5 particles after treatment with concentrated $\mathrm{NaOH}$ solution. 
RASĀYAN J. Chem.

Vol. 12 | No. 2 |939 - 946| April - June | 2019

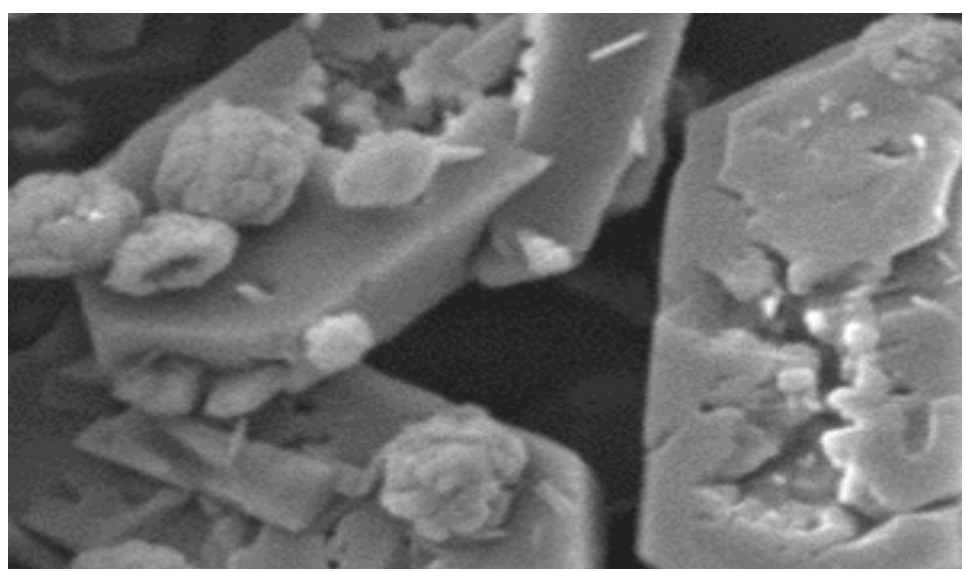

Fig.-3: Micrograph SEM of Commercial ZSM-5

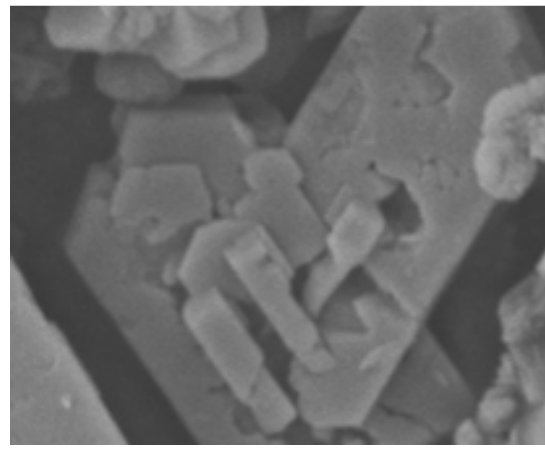

(a)

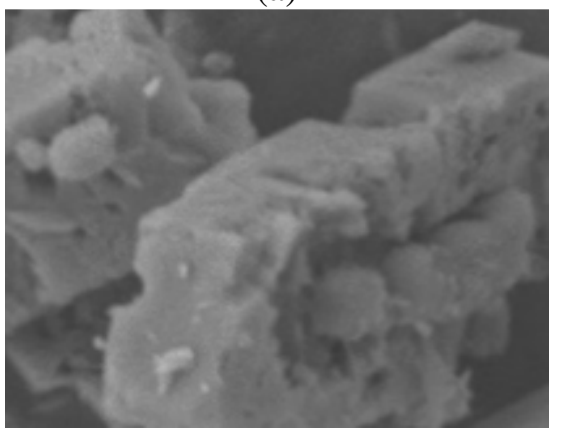

(c)

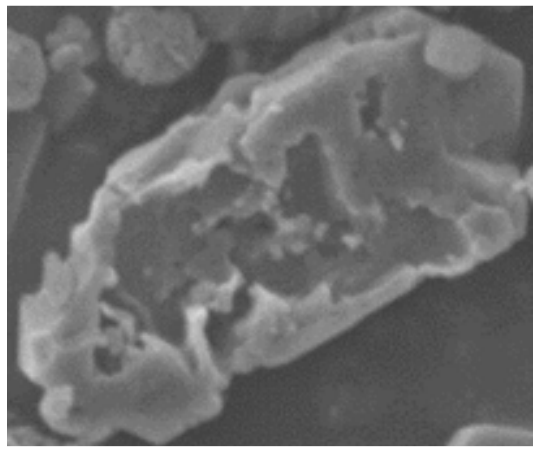

(b)

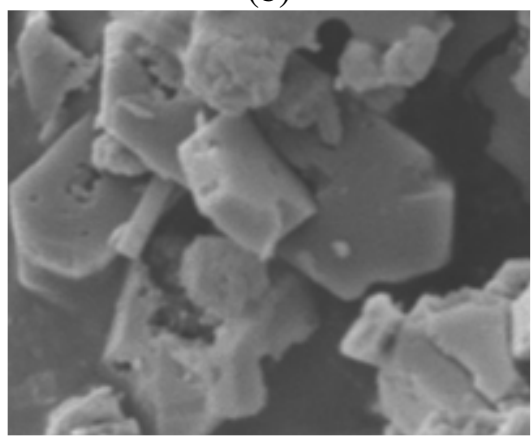

(d)

Fig.-4: Micrograph SEM of Hierarchical ZSM-5- (a) 0.25; (b) 0.5; (c) 1 and (d) 2

The reduction of surface area due to the formation of mesopore from micropore is confirmed by the presence of a hysteresis loop in the curves. It is clear that Fig.-5 shows the existence of micropores as indicated by the increasing curve at $\mathrm{p} / \mathrm{p}_{0}<0.1$ in all samples. This data confirms that conventional ZSM-5 has micropores in their particles. Furthermore, there is also mesopore as indicated by the presence of a hysteresis loop in all samples except ZSM-5-2.

The hysteresis loop is a characteristic of Type IV isothermal curves. It is caused by capillary condensation in the mesopore at high pressure ${ }^{6,16}$. The hysteresis loop also shows a difference in the amount of gas adsorbed and desorbed at the same relative pressure. Several curves show the increasing in mesopore characteristics as indicated by the widening of the hysteresis loop. In ZSM-5-0.25, 0.5 and 1, there is wider hysteresis loop which shows increasing the number of mesopores ${ }^{17}$. ZSM-5-2 only shows slight hysteresis loops. However, it also shows the increasing of adsorption at p/p0 $>0.9$ indicating the possibility of macropores formation. This BET data revealed that the treatment of ZSM-5 using NaOH solution could increase the mesoporosity of the ZSM- $5^{11}$. However, at high concentration, such as $0.5 \mathrm{M}$, the mesoporosity might be tremendously reduced forming macropores. 
RASĀYAN J. Chem.

Vol. 12 | No. 2 |939 - 946| April - June | 2019

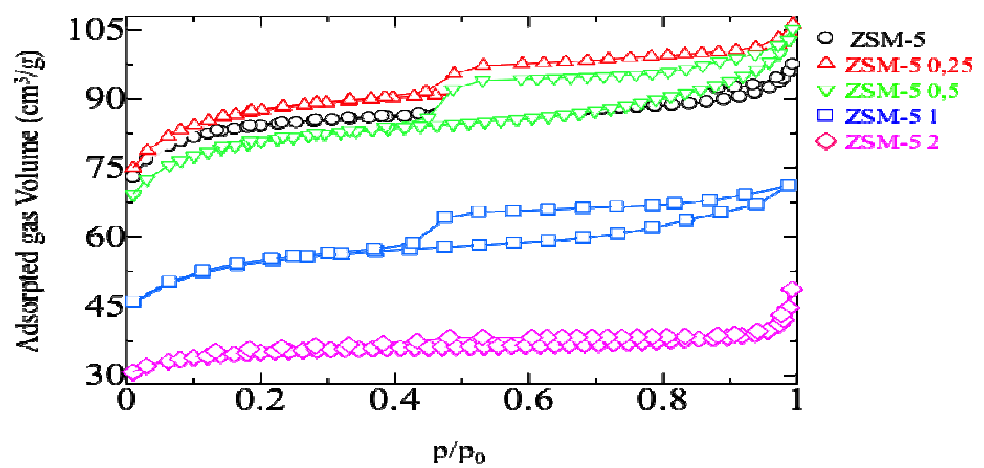

Fig.-5: Isothermal Curve of $\mathrm{N}_{2}$-Sorption from Commercial and Hierarchical ZSM-5

Table-4: Isotermal $\mathrm{N}_{2}$-sorption Analysis Data

\begin{tabular}{c|c|c|c|c}
\hline \multirow{2}{*}{ Material } & \multirow{2}{*}{ SBET $\left(\mathrm{m}^{2} / \mathrm{g}\right)$} & \multicolumn{2}{|c|}{ Pore Volume $(\mathrm{V})$} & \multirow{2}{*}{$\begin{array}{c}\text { Average Diameter } \\
\text { Pore }(\AA)\end{array}$} \\
\cline { 3 - 4 } & & V Micro $\left(\mathrm{cm}^{3} / \mathrm{g}\right)$ & V Meso $\left(\mathrm{cm}^{3} / \mathrm{g}\right)$ & 18.606 \\
\hline ZSM-5 & 324.762 & 0.108 & 0.042 & 19.701 \\
\hline ZSM-5-0.25 & 334.185 & 0.108 & 0.056 & 21.191 \\
\hline ZSM-5-0.5 & 307.497 & 0.097 & 0.065 & 25.436 \\
\hline ZSM-5-1 & 173.068 & 0.062 & 0.047 & 22.338 \\
\hline ZSM-5-2 & 134.929 & 0.044 & 0.031 & \\
\hline
\end{tabular}

Pore size distribution analysis is carried out using the $\mathrm{BJH}$ method to determine the pore diameter. The $\mathrm{BJH}$ method is used for pore distribution analysis for meso-sized poreonly. Figure-6 shows the pore size distribution $(\mathrm{BJH})$ with the largest population of pore diameter at 36-38 $\AA$. Increasing population or uniformity of pore diameter with diameters between $36-38 \AA$ occurs in ZSM-5-0.25 and ZSM-5-0.5. However, the ZSM-5-0.25 with mesopore diameter greater than $36-38 \AA$ is not formed.

Decreasing of micropore volume occurs with a higher concentration of $\mathrm{NaOH}$ as presented in Table- 4 . The micro-volume decreases due to the replacement of micropores with mesopores as desilication results. This is evidenced by the increasing of mesopore volume at ZSM-5-0.25, 0.5 and 1.0. Mesopores can grow from micropores which are increasingly enlarged to form mesopores. In addition, the decrease in mesopore volume occurs in ZSM-5-1 and ZSM-5-2 due to damage to ZSM-5 crystal structure ${ }^{6,11}$. The decreasing of mesopore volume in ZSM-5-2 is also predicted as the result of the formation of macropores as shown by BET curves.

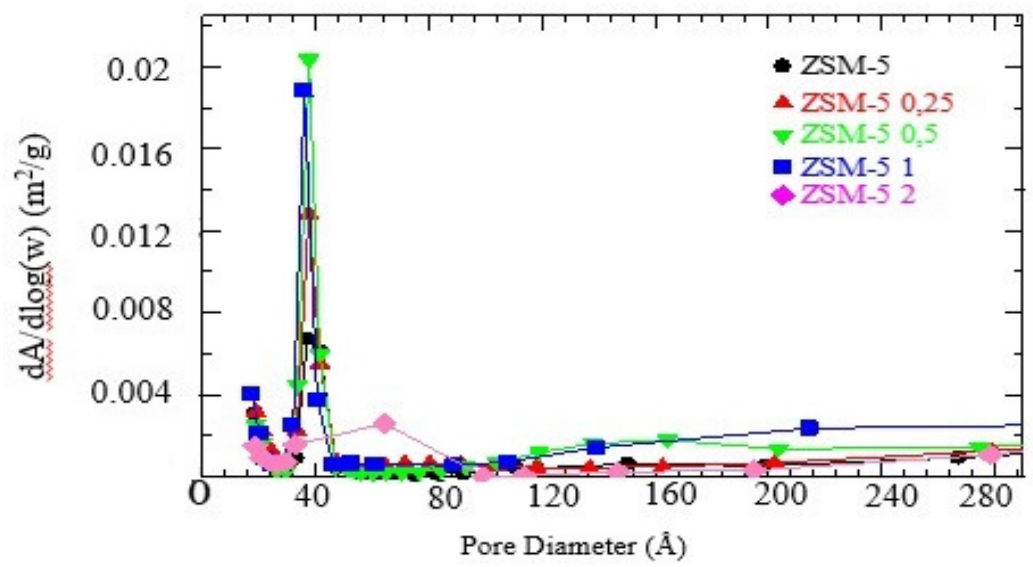

Fig.-6: Pore Size Distribution (BJH) Commercial and Hierarchical ZSM-5

\section{CONCLUSION}

The commercial and hierarchical ZSM-5 show that all samples consisted of ZSM-5 and tridymite phase as an impurity. The desilication process did not change in the crystal structure, but the crystallinity of ZSM-5 underwent changes where the highest crystallinity was obtained at $\mathrm{NaOH} 0.25 \mathrm{M}$. The difference in $\mathrm{NaOH}$ concentration did not cause the formation of new functional groups in ZSM-5 as shown by FTIR analysis. In addition, the increasing $\mathrm{NaOH}$ concentration also caused severe deterioration of ZSM-5 
RASĀYAN J. Chem.

Vol. 12 | No. 2 |939 - 946| April - June | 2019

particles. Analysis with isothermal $\mathrm{N}_{2}$-sorption shows that the surface area of the particle decreases as increasing of $\mathrm{NaOH}$ concentration. The $\mathrm{NaOH}$ treatment also succeeds to form mesopore in the particles as indicated by the presence of hysteresis loop. However, at high concentration, the mesopore is tremendously reduced forming macropore. The pore volume of the mesopore at the particles relies on 36$38 \AA$.

\section{ACKNOWLEDGMENT}

The author acknowledgesthe Ministry of Research, Technology and Higher Education (Kemenristekdikti) of Republic of Indonesia for funding this research in the under DRPM program fiscal year 2017 and LPPM-ITS for the financial support under the scheme of Hibah PUPT 2018.

\section{REFERENCES}

1. T.Xue, L. Chen, Y. M. Wang and M.Y. He, Microporous and Mesoporous Materials, 156, 97(2012), DOI:10.1016/j.micromeso.2012.02.022

2. P. Sagar, V. Sharma, and R. Kumar, Rasayan Journal of Chemistry, 3, 1(2018).

3. J. C. Moreno-Piraján andL. Giraldo, Rasayan Journal of Chemistry,6, 3(2016).

4. S. Abelló, A. Bonilla, and J. Pérez-Ramírez, Applied Catalysis A: General, 364, 1(2009), DOI:10.1016/j.apcata.2009.05.055

5. S. Mitchell, A. B. Pinar, J. Kenvin, P. Crivelli, J. Kärger, and J. Pérez-Ramírez, Nature Communications, 6, 1(2015), DOI:10.1038/ncomms9633.

6. J. Jung, C. Jo, F. M. Mota, J. Cho, and R. Ryoo, Applied Catalysis A: General, 492, 68(2015),DOI:10.1016/j.apcata.2014.12.019.

7. D. P. Gamliel, H.J. Cho, W. Fan, and J. A. Valla, Applied Catalysis A: General, 522, 109(2016), DOI: $10.1016 /$ j.apcata.2016.04.026

8. J. Van Aelst, D. Verboekend, A. Philippaerts, N. Nuttens, M. Kurttepeli, E. Gobechiya, M. Haouas, S. P. Sree, J. F.M. Denayer, andJ. A. Martens, Advanced Functional Materials, 25, 7130(2015), DOI:10.1002/adfm.201502772

9. C. Selzer, T. Biemelt, A. Werner, and S. Kaskel, Microporous and Mesoporous Materials, 261, 51(2018), DOI:10.1016/j.micromeso.2017.11.005

10. P. Matias, C. Sá Couto, I. Graça, J. M. Lopes, A. P. Carvalho, F. Ramôa Ribeiro andM. Guisnet, Applied Catalysis A: General, 399, 100(2011), DOI:10.1016/j.apcata.2011.03.049

11. K. Sadowska, K. Góra-Marek, M. Drozdek, P. Kuśtrowski, J. Datka, J. M. Triguero, and F. Rey, Microporous and Mesoporous Materials, 168, 195(2013), DOI:10.1016/j.micromeso.2012.09.033

12. J. Li, X. Li, G. Zhou, W. Wang, C. Wang, S. Komarneni, and Y. Wang, Applied Catalysis A: General, 470, 115(2014), DOI:10.1016/j.apcata.2013.10.040

13. C. Mei, Z. Liu, P. Wen, Z. Xie, W. Hua, and Z. Gao, Journal of Materials Chemistry, 18, 29(2008), DOI: $10.1039 / \mathrm{b} 805385 \mathrm{e}$

14. M. Rahmani andM. Taghizadeh, Reaction Kinetics, Mechanisms and Catalysis, 122, 1(2017), DOI: $10.1007 / \mathrm{s} 11144-017-1204-0$

15. R. Khoshbin and R. Karimzadeh, Advanced Powder Technology, 28, 8(2017), DOI:10.1016/j.apt.2017.04.024

16. K. Menad, A. Feddag, and K. Rubenis, Rasayan Journal of Chemistry, 9, 4(2016).

17. D. Hartanto, Doctoral Thesis, Department of Chemistry, Institut Teknologi Sepuluh Nopember, Surabaya, Indonesia, (2016).

[RJC-5142/2018] 